\title{
Effect of contrast level and temporal order on the Ebbinghaus circles illusion
}

\author{
TED JAEGER and ROBERT H. POLLACK \\ University of Georgia, Athens, Georgia 30602
}

\begin{abstract}
Forty-eight undergraduates between 17 and 24 years of age judged the size of the central circle in Ebbinghaus figures presented under either simultaneous or successive viewing conditions. Successive presentation diminished the magnitude of illusion produced by the underestimated Ebbinghaus figure and totally eliminated the illusion normally produced by the overestimated figure. Variations in lightness contrast of the central and surrounding circles significantly altered the magnitude of illusion produced by both simultaneous Ebbinghaus figures as well as that produced by the successive underestimated figure. These data suggest the presence of a contour interaction process in both simultaneous Ebbinghaus illusions.
\end{abstract}

The Ebbinghaus, or Titchner, circles illusion has typically been attributed to the operation of a cognitive size contrast mechanism (Cooper \& Weintraub, 1970; Coren \& Miller, 1974; Piaget, 1969; Sanford, 1898). Briefly, this account of the illusion suggests that judgments of the size of the central circle in an Ebbinghaus figure are made in relation to the size of the surrounding circles. When large surrounding circles are present, the contrast mechanism causes the central circle to appear smaller than its physical size; when small surrounding circles are present, the contrast mechanism causes the central circle to appear larger than its physical size. Massaro and Anderson (1971) have quantified this analysis of the Ebbinghaus illusion and have presented evidence to indicate that the magnitude of the contrast effect varies directly with the number of surrounding circles and inversely with their separation from the central circle.

Recently, however, Girgus, Coren, and Agdern (1972) have suggested that a Delboeuf-like contour interaction process must be at least partially responsible for the Ebbinghaus illusion. This contention was supported by demonstrating that increasing the separation of the central and surrounding circles of both Ebbinghaus illusion figures resulted in a decrease in the apparent size of the central circle. Furthermore, it was found that large separations of the central and surrounding circles reversed the direction of the normally overestimated illusion. Both findings are inconsistent with a size contrast interpretation of the illusion and suggest that the ring of surrounding circles may be functioning in a manner analogous to the outer concentric circle of the Delboeuf figure.

Requests for reprints should be sent to Robert H. Pollack, Department of Psychology, University of Georgia, Athens, Georgia 30602.
If Girgus et al. (1972) are correct in assuming that a Delboeuf-like contour interaction process is partially responsible for the Ebbinghaus illusion, corresponding alterations in stimulus variables should effect both the Ebbinghaus and Delboeuf illusions similarly. Oyama (1962) and Weintraub, Wilson, Greene, and Palmquist (1969) have shown that decreasing the lightness contrast of the outer circle of the Delboeuf figure decreases illusion magnitude while decreasing the lightness contrast of the inner circle increases illusion magnitude. Although lightness is a potent variable in determining magnitude of the Delboeuf illusion when inducing and test components of the figure are presented simultaneously, it appears to be ineffective if inducing and test components are presented successively (Oyama, 1960; Sjostrom \& Pollack, 1971). Nevertheless, successive presentation does diminish the apparent size of the central circle of the Delboeuf figure, producing a reversed illusion (Ikeda \& Obani, 1955; Adam, 1966). In order to delineate further the similarities between the Delboeuf and Ebbinghaus illusions, the present experiment was designed to investigate the effects of lightness contrast and presentation sequence on the magnitude of the two Ebbinghaus illusions.

\section{METHOD}

\section{Subjects}

The subjects were 24 male and 24 female white undergraduate students ranging in age from 17 to 24 years. Each had visual acuities, corrected or uncorrected, of $20 / 20$ or better, as measured with a Snellen chart.

\section{Stimuli}

All stimulus figures were drawn on white $5 \times 7$ in. file cards with black (2.7\% reflectance) and light gray ( $68.4 \%$ reflectance) India ink. The diameter of the central circle in all Ebbinghaus figures was $8.88 \mathrm{~mm}$. The diameter of the four small surrounding circles was $3.0 \mathrm{~mm}$, while the diameter of the four large sur- 
rounding circles was $14.76 \mathrm{~mm}$. The separation between the central circle and the proximal edges of either the large or small surrounding circles was $2 \mathrm{~mm}$.

The stimulus cards used for the simultaneous viewing conditions contained an entire Ebbinghaus figure and a comparison circle. The Ebbinghaus figures were drawn with either black surrounding circles and a gray central circle (BG), black surrounding and central circles (BB), gray surrounding and central circles (GG), or gray surrounding circles and a black central circle (GB). The lightness contrast of the comparison circle always matched that of the central circle of the Ebbinghaus figure. On each of the stimulus cards, the Ebbinghaus figure was centered $22 \mathrm{~mm}$ to one side of a central fixation point and a comparison circle was centered an equal distance to the opposite side. For the overestimated Ebbinghaus figure, the diameter of the comparison circles ranged from 7.63 to $10.63 \mathrm{~mm}$ in .25 -mm-interval steps, while for the underestimated Ebbinghaus figure, the diameter of the comparison circles ranged from 7.38 to $10.38 \mathrm{~mm}$, also in .25-mm-interval steps. The maximal height of the stimulus array containing the underestimated Ebbinghaus figures was $1^{\circ} 44^{\prime}$, while width varied from $3^{\circ} 10^{\prime} 10^{\prime \prime}$ to $3^{\circ} 14^{\prime} 20^{\prime \prime}$. For the overestimated Ebbinghaus figures, the maximal height of the stimulus array was $1^{\circ} 35^{\prime}$ and the width varied from $2^{\circ} 36^{\prime} 10^{\prime \prime}$ to $2^{\circ} 41^{\prime} 20^{\prime \prime}$.

For successive viewing conditions, eight stimulus cards were constructed containing only the surrounding circles of the Ebbinghaus figure. Four of the cards contained large surrounding circles drawn with either black or light gray India ink and centered $22 \mathrm{~mm}$ to either the right or left of a central fixation point. The other four cards contained small surrounding circles again drawn with either black or light gray India ink and centered $22 \mathrm{~mm}$ to either the right or left of a central fixation point. The central and comparison circles were drawn on a separate set of 24 cards. Half of these cards contained black central circles and a comparison circle, which ranged in diameter from 7.38 to $10.38 \mathrm{~mm}$ in .25 -mm-interval steps. The other half contained gray central circles and a comparison circle, which also ranged in diameter from 7.38 to $10.38 \mathrm{~mm}$ in .25 -mm-interval steps. The central circles were centered $22 \mathrm{~mm}$ to either the right or left of a central fixation point, and the comparison circle was centered an equal distance to the opposite side. By pairing the stimulus card containing only the surrounding circles with the appropriate stimulus card containing only the central and comparison circles, successive Ebbinghaus illusion figures were produced with black surrounding circles and a gray central circle (BG), black surrounding and central circles (BB), gray surrounding and central circles (GG), and gray surrounding circles and a black central circle (GB). The visual angle sizes of the stimulus arrays containing the successive Ebbinghaus figures matched those of the simultaneous figures.

In addition to the illusion stimuli, control stimuli were constructed consisting of a black circle $8.88 \mathrm{~mm}$ in diameter centered $22 \mathrm{~mm}$ to either the right or left of a central fixation point and a black comparison circle centered an equal distance to the opposite side. The diameter of the comparison circle ranged from 7.88 to $9.88 \mathrm{~mm}$ in .25 -mm-interval steps. The maximal height of this stimulus array was $26^{\prime} 40^{\prime \prime}$, and the width varied from $2^{\circ} 24^{\prime}$ to $2^{\circ} 29^{\prime} 20^{\prime \prime}$.

\section{Procedure}

The subjects were randomly assigned to one of four experimental groups with the stipulation that an equal number of males and females be represented in each group. Group 1 saw the overestimated Ebbinghaus figure under conditions of simultaneous presentation, Group 2 saw the overestimated Ebbinghaus figure under conditions of successive presentation, Group 3 saw the underestimated Ebbinghaus figure under conditions of simultaneous presentation, and Group 4 saw the underestimated Ebbinghaus figure under conditions of successive presentation.

Before the experimental session, subjects were dark-adapted for 5 min, during which time instructions were given. Each subject was told to respond "larger"' when the comparison circle appeared larger than the central circle of the Ebbinghaus figure and "smaller" when the comparison circle appeared smaller than the central circle of the Ebbinghaus figure. No "equal to" responses were accepted.

Stimuli were presented in a three-channel tachistoscope (Scientific Prototype, Model GB). The level of illumination at the eyepiece as measured by a MacBeth illuminometer was $16.538 \mathrm{~mL}$. Each experimental session began and ended with a control condition in which subjects estimated the size of a test circle of the same diameter as the central circle in the Ebbinghaus figures. The control condition contained four trials, each trial consisting of eight judgments. The order of stimulus presentation was determined by a converging method of limits (Clem \& Pollack, 1975), and initiation of each trial with either a large or small comparison circle was counterbalanced with an ABBA design. Also, an ABBA design was used to counterbalance the placement of the test and comparison circles to either the right or left of the fixation point.

After the control trials, the subjects continued, in their respective experimental groups, judging the size of the central circle in one Ebbinghaus illusion figure under either simultaneous or successive viewing conditions. Repeated measures were taken across lightness conditions, with each subject receiving 8 trials per lightness condition and 12 judgments per trial. Again, order of stimulus presentation was determined by a converging method of limits, and initiation of trials with either a large or small comparison circle was counterbalanced with an ABBA design. The placement of the Ebbinghaus figure and comparison circle to either the right or the left of the fixation point was also counterbalanced, following an ABBA design, and a balanced square design was used to determine the order of presentation of lightness conditions for each subject.

\section{RESULTS}

PSEs, defined as the point at which a changed response was maintained for three consecutive judgments, were calculated for each subject on each trial. These scores were then converted to illusion magnitude scores by subtracting the control PSE from the mean PSE of each experimental condition for each subject. The mean illusion magnitude score in each of the 16 experimental conditions is presented in Figure 1. Observation of the figure reveals that the central circle of the Ebbinghaus figure with small surrounding circles was overestimated, and the central circle of the Ebbinghaus figure with large surrounding circles was underestimated. Successive presentation diminishes the magnitude of the underestimated illusion while leaving no appreciable illusion in the normally overestimated figure. Results of four criterion t tests, one for each lightness condition, comparing the magnitude of the normally overestimated successive illusion to zero were all insignificant.

The data presented in Figure 1 were analyzed with a three-way mixed-model analysis of variance. Significant main effects were obtained for illusion $(F=$ 52.812 , df $=1,44, \mathrm{p}<.001)$ and lightness condition $(\mathrm{F}=15.571, \mathrm{df}=3,132, \mathrm{p}<.001)$. The main effect of presentation was not significant $(F=.754$, $\mathrm{df}=1,44, \mathrm{p}>.20$ ) because it is averaged out as a consequence of collapsing across illusion conditions. 


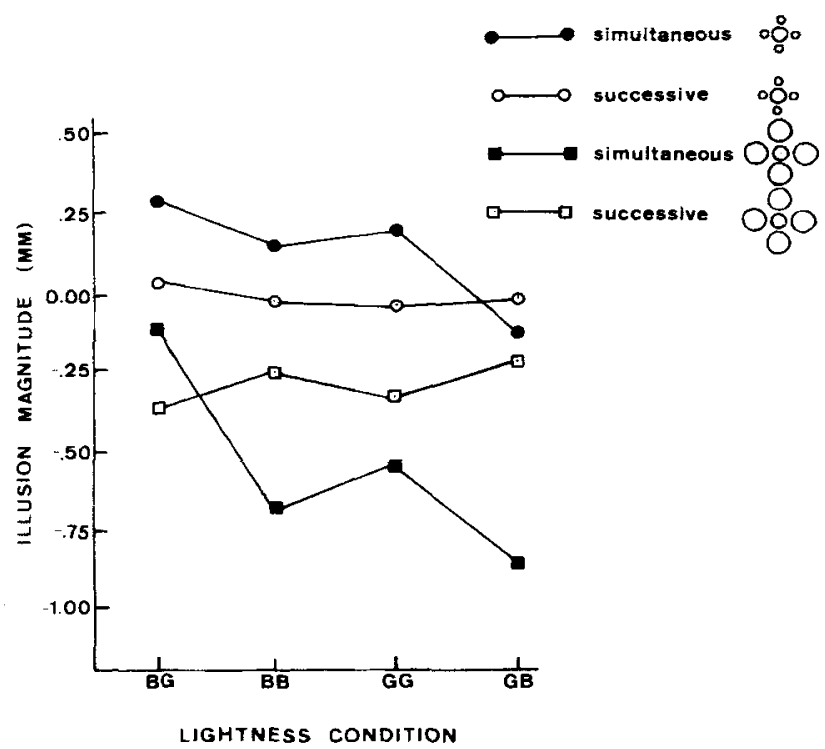

Figure 1. Illusion magnitude as a function of lightness condition. Each data point is averaged across 12 subjects with eight converging method of limits trials per subject.

In addition to these main effects, significant twoway interactions were obtained for Illusion by Presentation $(F=6.875$, df $=1,44, p<.05)$ and Lightness by Presentation $(F=23.114$, df $=3,132$, $\mathrm{p}<.001)$. The Illusion by Presentation interaction reflects the fact that for the overestimated illusion successive presentation diminishes the apparent size of the central circle, while for the underestimated illusion successive presentation increases the apparent size of the central circles. This interaction was further evaluated by conducting tests of the simple effects of presentation at each illusion level. For the overestimated Ebbinghaus illusion, the simple effect of presentation was not significant $(\mathrm{F}=1.512, \mathrm{df}=1,44, \mathrm{p}>.20)$, but for the underestimated illusion, the simple effect of presentation was significant $(\mathrm{F}=6.054, \mathrm{df}=1,44, \mathrm{p}<.05)$. The Lightness by Presentation interaction appears to result from the larger effect of lightness on the simultaneous illusions than on the successive illusions. This observation is confirmed by two tests of simple effects which reveal that the effect of lightness is significant under simultaneous presentation condition $(F=114.31, \mathrm{df}=3,132, \mathrm{p}<.001)$ but not under successive presentation conditions $(F=1.77$, df $=3,132, p>.20)$.

Finally, the three-way interaction of Lightness by Presentation by Illusion, was also significant $(F=$ 5.885 , df $=3,132, p<.01)$. In order to determine the source of this triple interaction, the data were divided by presentation condition and two two-way mixed-model analyses of variance were performed. In the first, the effect of lightness on the simultaneous illusions was examined, and it was found, as expected, that the main effects of lightness $(F=27.20$, df $=$ $3,66, p<.001)$ and illusion $(F=49.438$, df $=1,22$, $\mathrm{p}<.001)$ were significant. More interestingly, the Lightness by Illusion interaction was also significant $(\mathrm{F}=4.55, \mathrm{df}=3.66, \mathrm{p}<.01)$, indicating that the lightness effect was greater for the underestimated than for the overestimated illusion. In the second two-way mixed-model analyses of variance, the effect of lightness on the successive illusions was investigated. The main effect of illusion was significant $(\mathrm{F}=10.348, \mathrm{df}=1,33, \mathrm{p}<.01)$, but the main effect of lightness did not reach significance $(F=$ 1.05 , df $=3,66, p>.20$ ). However, the Lightness by Illusion interaction was again significant $(F=$ 3.15 , df $=3,66, p<.05$ ), indicating that the magnitude of the lightness effect differed for the two successive illusions. Since this main effect of lightness failed to reach statistical significance and the Lightness by Illusion interaction was significant, tests of the simple effects of lightness on each of the two successive illusions were conducted. For the normally overestimated successive illusion, the simple effect of lightness was not significant $(F=$ 1.062 , df $=3,132, \mathrm{p}>.20$ ), but for the underestimated successive illusion, the effect of lightness was significant ( $F=6.085$, df $=3,132, p<.01)$. Thus, the triple interaction of Lightness by Presentation by Illusion results from the differential effects of lightness at each presentation level of each illusion.

\section{DISCUSSION}

The data reported here indicate a close relationship between the overestimated Ebbinghaus and Delboeuf illusions. As with the Delboeuf illusion, successive presentation of inducing and test components results in a reduction in the apparent size of the central circle. Although the illusion produced by the overestimated successive Ebbinghaus figure is not significantly reversed, the apparent size of the central circle in three of the four lightness conditions is less than the apparent size of the control circle. If the ring of surrounding circles functions analogously to the outer circle of the Delboeuf figure, it would be expected that increasing the number of surrounding circles would produce a larger underestimation in central circle size. Our next experiment will deal directly with this problem. In any case, the failure of the overestimated Ebbinghaus illusion to persist under conditions of successive presentation argues against an explanation of this illusion based on a cognitive size contrast mechanism. Furthermore, the effectiveness of the lightness variables in altering the simultaneous illusion magnitude suggests the presence of a contour interaction process. As with the Delboeuf illusion, reducing the lightness contrast of the inducing component relative 
to that of the test component decreases illusion magnitude. Conversely, decreasing the lightness contrast of the test component relative to that of the inducing component increases illusion magnitude. While the effect of lightness is not significant for the successive overestimated illusion, analogous reports of the absence of a lightness effect on the successive Delboeuf are extant (Oyama, 1960; Sjostrom \& Pollack, 1971). Taken together, then, these data provide support for the notion that the same contour interaction process may underlie both the overestimated Ebbinghaus and Delboeuf illusions.

Turning to the underestimated Ebbinghaus figure, a different pattern of results emerges. While the effect of lightness on the simultaneous figure is again significant, suggesting the presence of a contour interaction process, this effect is systematically reversed when compared to the overestimated simultaneous illusion. This reversed effect seems to indicate that both a contour interaction and a cognitive contrast process may be responsible for the underestimated illusion. Since a Delboeuf-like contour interaction process would produce an attraction effect and a consequent enlargement of the central circle, this mechanism would work against the size contrast effect induced by the large surrounding circles. The fact that the smallest simultaneous underestimated Ebbinghaus illusion was obtained with high-contrast surrounding circles and a low-contrast central circle seems to indicate that the contrast effect was weakened by the contour interaction process.

With respect to the successive underestimated illusion, the significant effect of lightness was unexpected. The finding that variations in the lightness contrast of inducing contours can significantly alter the magnitude of successive illusion is inconsistent with the findings of Oyama (1960) and Sjostram and Pollack (1971). It should be noted that despite the statistical significance of these results, the effect of lightness on the successive underestimated illusion was quite small compared to the lightness effect obtained with the simultaneous illusions. Consequently, at present, we prefer not to attach any psychological importance to this finding. However, it is clear that successive presentation of inducing and test components of this figure does not eliminate the illusion. The persistence of the illusion under successive viewing conditions provides further evidence that the underestimated simultaneous illusion is partially attributable to the operation of a size contrast mechanism.

Finally, it should be noted that the findings reported here have implications for the developmental classification of the Ebbinghaus illusions. These illusions have been reported to increase in magnitude through childhood (Comalli, 1965; Wapner \& Werner, 1957; Wapner, Werner, \& Comalli, 1960) and have therefore been considered Type II phenomena. However, Pollack (1969) has provided evidence that illusions generated by a contour interaction process typically show Type I age trends, i.e., decrease in illusions magnitude with increasing chronological age. Since the present experiment has adduced evidence for a contour interaction process in both Ebbinghaus illusions, the classification of these illusions as Type II phenomena is questioned. The aforementioned ontogenetic studies of the Ebbinghaus illusions have employed large stimulus figures and unlimited exposure durations, thus confounding the effects of scanning, attention, and temporal integration with their results. An age trend study with the Ebbinghaus figures and stimulus conditions employed here should clarify the interrelationship and relative strength of the contour interaction and cognitive contrast processes responsible for the Ebbinghaus illusions. Such a study is planned.

\section{REFERENCES}

AdAM. J. The relationship between visual illusions and figural aftereffects. A ustralian Journal of Psychology, 1966, 18, 130-136.

Clem, R. K., \& Pollack, R. H. Illusion magnitude as a function of visual field exposure. Perception \& Psychophysics. 1975. 17. 450-454.

Comalli, P. E. Cognitive functioning in a group of $80-90$ year old men. Journal of Gerontology, 1965, 20, 14-17.

Cooper, L. A., \& Weintraub, P. J. Delboeuf-type circle illusions: Interactions among luminance. temporal characteristics and inducing figure variations. Journal of Experimental Psychology. $1970,85,75-82$.

Coren, S., \& Miller, J. Size contrast as a function of figural similarity. Perception \& Psychophysics, 1974, 16, 355-357.

Girgus, J. S., Coren, S., \& Agdern. M. The interrelationship between the Ebbinghaus and Delboeuf illusions. Joumal of Experimental Psychology, 1972, 95, 453-455.

IKeDA, H., \& ObanaI, T. Studies in figural aftereffects (IV): The contrast confluence illusion of concentric circles and the figural aftereffect. Japanese Psychological Research, 1955, 2, 17-23.

MAssaro. D. W., \& ANDERSON, N. H. Judgmental model of the Ebbinghaus illusion. Journal of Experimental Psychology. 1971. 89, 147-151.

OY ама. T. Figural aftereffects as a function of hue and brightness. Japanese Psychological Research, 1960, 2, 74-80.

Oy ама, T. The effect of hue and brightness on the size illusion of concentric circles. American Journal of Psychology, 1962, 75, 45-55.

Praget, J. The mechanisms of perception. New York: Basic Books, 1969.

Pollack, R. H. Some implications of ontogenetic changes in perception. In D. Elkind \& J. H. Flawell (Eds.), Studies in cognitive development: Essay in honor of Jean Piaget. New York: Oxford University Press, 1969.

SANFORD, E. C. A course in experimental psychology. Part I: Sensation and perception. Boston: Heath, 1898.

Sjostram, K. P., \& Pollack, R. H. The effect of simulated receptor aging on two types of visual illusions. Psychonomic Science, 1971, 23, 147-148. 
Wapner. S. \& Werner, H. Perceptud delielopment. Worcester, Mass: Chark University Press. 1957.

Wapner. S., Werner, H. \& Comalli, P. E. Perception of parthole relationships in middle and old age. Joumal of Gerontology, 1960. 15. $+12-410$.

Weintraci, D. J.. Wilson, B. A.. Greene, R. D., \&
PalmQlist, M. J. Delboeuf illusion: Displacement versus diameter, arc deletions, and brightness contrast. Journal of Experimental Psychology, 1969, 80, 505-511.

(Received for publication August 2, 1976: revision accepted November 11, 1976.) 